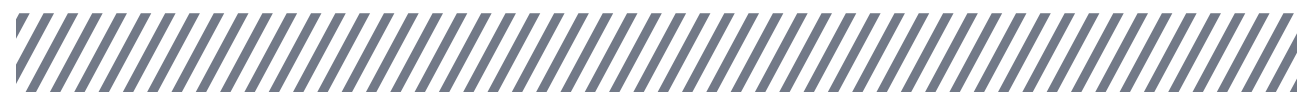



Dr. Dieter Höfler

Gründer und

Geschäftsführer

Tectos $\mathrm{GmbH}$

\section{Testing bietet enorme Möglichkeiten}

Unsicherheit hat sich breitgemacht. Schon vor Corona war es unruhig in der Automobilindustrie. Und je länger die durch den Lockdown verstärkte Krise andauert, desto kontroverser wird diskutiert. Folgt man der politischen Diskussion, so ist die E-Mobilität der Heilsbringer und der Verbrennungsmotor wird verteufelt.

Ist dies der richtige Ansatz oder sollten wir - als kreative Menschen - nicht frei und nach allen Richtungen offen sein?

Ist diese essenzielle Krise einer über Jahrzehnte erfolgsverwöhnten Industrie nicht auch eine Chance, sich wieder auf die Grundpfeiler zu besinnen - nämlich Produkt, Mobilität und die realen Verbindungen zwischen Menschen, also abseits der sozialen Netzwerke?

Wir sagen ja. Die Möglichkeiten, die sich gerade für Unternehmen im Bereich Testing bieten, sind enorm. Wir sind gefordert, unsere Kunden beim Transformationsprozess in eine ökologisch saubere Zukunft zu begleiten. Offen, ohne Vorurteile, dafür aber immer den Blick auf das Wesentliche gerichtet und gleichzeitig Chancen zu sehen, die sich für unsere Kunden dadurch ergeben.

Dazu müssen wir in der Lage sein, selbst diese Veränderung zu tragen, sowohl technisch als auch in den eigenen persönlichen Einstellungen.
Ein Beispiel: Die Corona-Krise hat uns gezeigt, dass Heimarbeit und die Kommunikation über Videokonferenzen Meetings effizienter machen. Gleichzeitig hat dieser Arbeitsstil auch klar die Grenzen aufgezeigt: Ohne zwischenmenschlichen persönlichen Kontakt ist der kreative Entwicklungsprozess schwierig. Denn gerade das gedankliche Ping-Pong-Spiel zwischen den Kollegen geht dadurch verloren.

Werden durch diese Erfahrungen die richtigen Schlüsse gezogen und in die Unternehmenskultur integriert, motiviert dies Mitarbeiter zu Höchstleistungen und lässt gleichzeitig freie Ressourcen entstehen, die wiederum in neue Lösungen und Ideen kanalisiert werden.

Wir haben diese Ressourcen zu einer genauen Analyse möglicher Zukunftsszenarien genutzt und kamen zu folgendem Schluss: Wir setzen auf Pluralität der Antriebskonzepte Wasserstoff, synthetische Kraftstoffe sowie Batterien. Dabei ist unser Anspruch, das Beste aus allen Welten zu vereinen, um so das Optimum zu erzielen. Nicht nur für unsere Kunden, sondern auch für die Kunden unserer Kunden.

Denn seien wir ehrlich: Letztendlich zählt der Konsument, der durch die Investition in ein neues Fahrzeug das Produkt unserer Kunden und damit gleichzeitig unsere Ideen und unsere Arbeit honoriert. 